\title{
Políticas públicas de promoção à saúde de crianças e adolescentes nas escolas
}

\author{
Public policies to promote the health of children and adolescents in \\ schools
}

Políticas públicas para promover la salud de niños y adolescentes en las escuelas

Maurício Osório Gonçalves¹; Renan Antônio da Silva²; Georgia Rebouças ${ }^{3}$

\section{RESUMO}

O artigo apresenta como a mudança de paradigma nas ações de saúde, quando passa de um modelo focado na cura para uma abordagem centrada na promoção de saúde, influenciou a forma de implementação das políticas públicas de promoção da saúde às crianças e adolescentes. Nesse sentido, tem destaque as recomendações de organismos internacionais e nacionais quanto a implementação intersetorial e a inclusão de novos atores e instituições não-governamentais nas ações de políticas públicas de saúde.

Palavras-chave: Políticas públicas intersetoriais; Promoção da saúde; Saúde escolar.

\begin{abstract}
The article presents how the paradigm shift in health actions, when shifting from a model focused on healing to an approach focused on health promotion, influenced the way in which public policies for health promotion were implemented for children and adolescents. In this sense, the recommendations of international and national organizations regarding the intersectoral implementation and the inclusion of new actors and nongovernmental institutions in the actions of public health policies are highlighted.
\end{abstract}

Keywords: Intersectoral public policies; Health promotion; School health.

\section{RESUMEN}

El artículo presenta cómo el cambio de paradigma en las acciones de salud, al pasar de un modelo centrado en la curación a un enfoque centrado en la promoción de la salud, influyó en la forma en que se implementaron las políticas públicas de promoción de la salud para la niñez y la adolescencia. En este sentido, se destacan las recomendaciones de organismos internacionales y nacionales en cuanto a la implementación intersectorial y la inclusión de nuevos actores e instituciones no gubernamentales en las acciones de las políticas públicas de salud.

Palabras clave: Políticas públicas intersectoriales; Promoción de la salud; Salud escolar.

\footnotetext{
${ }^{1}$ Mestre em Políticas Públicas pela Universidade Federal do ABC. Atualmente é consultor pela UNESCO. E-mail: mauricioogon@gmail.com

2 Professor Permanente no Programa de Pós - Graduação em Políticas Públicas da Universidade Estadual do Ceará (UECE), Fortaleza/CE - Brasil. Bolsista da FUNCAP. E-mail: r.silva@unesp.br

${ }^{3}$ Mestranda em Políticas Públicas na Universidade Estadual do Ceará (UECE), Fortaleza/CE - Brasil. E-mail: georgiareboucas@yahoo.com.br
} 


\section{INTRODUÇÃO}

Problemas de saúde em crianças e adolescentes podem ser considerados comuns e percebidos como parte da transformação que ocorre durante o seu desenvolvimento físico, motor e psicológico. Contudo, estudos de amplitude nacional tais como a Pesquisa Nacional de Saúde do Escolar e o Estudo de Riscos Cardiovasculares em Adolescentes apontam que há diversas comorbidades relacionadas ao excesso de peso, ao aumento de pressão arterial e ao colesterol alto, que tanto comprometem sua qualidade de vida como também podem contribuir para a redução na sua expectativa de vida.

Os estudos demonstram que hábitos de vida como o sedentarismo, a alta ingestão de alimentos industrializados, o consumo de álcool e drogas levam a estes problemas de saúde. A adoção de hábitos saudáveis por crianças e jovens, em sua ampla acepção, é uma recomendação feita a partir desses estudos. Cabe salientar a necessidade de que os resultados produzam mudanças em curto prazo com relação à disponibilidade e estímulo ao consumo de alimentos mais saudáveis, bem como ao estímulo a atividades e comportamentos que reduzam a exposição desses adolescentes à obesidade, ao sedentarismo e ao tabagismo, entre outros fatores de risco cardiovascular (BLOCH; CARDOSO; SICHIERI, 2016).

Nessa linha, tem sido desenvolvidas pesquisas de alcance internacional tratando do tema da saúde de crianças e adolescentes. Entre essas é possível mencionar o estudo "Growing up unequal: gender and socioeconomic differences in young people's health and well-being" publicado em 2016 como continuidade de uma série de estudos desenvolvidos pelo World Health Organization (WHO).

Ao longo de 30 anos, o WHO produz pesquisas intituladas "Health Behaviour in School-Aged Children (HBSC) Study" (WHO, 2016) com o objetivo de compreender os hábitos de saúde de crianças e adolescentes no contexto social, ou seja, nos lares, nas escolas, junto à família e amigos, buscando entender como esses fatores individualmente e em conjunto influenciam a saúde tanto de crianças e jovens quanto de adultos. Um dos apontamentos desse estudo evidencia a relevância de políticas públicas focadas no desenvolvimento de comportamentos saudáveis em idades precoces (WHO, 2016).

No contexto nacional, o Instituto Brasileiro de Geografia e Estatística (IBGE) publica desde 2009 a Pesquisa Nacional de Saúde do Escolar (PeNSE) com coleta de dados de hábitos de saúde de adolescente entre 13 a 17 anos frequentando as etapas do $6^{\circ}$ ao $9^{\circ}$ ano do ensino fundamental e a $1^{a}$ a $3^{a}$ série do ensino médio. Partindo da premissa da importância da escola como um lócus de formação, prevenção e monitoramento de fatores de risco à saúde, o estudo "evidencia a necessidade de acompanhamento da situação de saúde de adolescentes, objetivando prover informações que reflitam a complexidade e a dinâmica de mudanças a que está sujeito esse grupo etário" (IBGE, 2016).

O conceito de promoção da saúde como base de ação, é dos princípios desta concepção. Esta perspectiva abarca aspectos sociais, culturais, antropológicos, econômicos e problematiza como instituições, espaços sociais e atores podem também contribuir para promovê-la. Entre os princípios que permeiam a promoção da saúde encontra-se a intersetorialidade como um esforço de mobilizar estas instituições e atores. Nessa linha, a World Health Organization (WHO), no documento Health 2020, propôs metas para estruturar políticas de saúde e bem-estar, e no documento "Intersectoral Governance for Health in All Policies" apontou como imprescindível a compreensão da promoção da 
saúde e do bem-estar como conceitos e práticas que vão além da área da saúde e, portanto, para a necessidade de conexão entre as diversas políticas públicas.

Documento proposto pela WHO indica, assim, a importância de se organizar uma estrutura intersetorial que facilite a elaboração de mecanismos de colaboração entre os agentes e garanta uma visão ampliada dos serviços em saúde no processo formulação de políticas públicas em todas as áreas do governo. Quanto à perspectiva de políticas públicas intersetoriais de saúde e educação há uma mudança de paradigma na área da saúde quando ocorre um rompimento com a lógica setorial, que permite a criação de uma estrutura intersetorial. Esta última possibilita a integração de diversas áreas, atores e instituições com interesses diversos, para discutir e implementar ações de saúde. Esse movimento cria novas questões na estruturação e operacionalização das ações de saúde.

Entre as políticas públicas que tratam da questão da saúde da criança e adolescente em idade escolar há o Programa Saúde na Escola (PSE), instituído pelo decreto presidencial no 6.286, de 05 de dezembro de 2007. O Programa integra o Plano Nacional de Atenção Básica, plano este pertencente às ações do Ministério da Saúde acompanhado e coordenado pelo Departamento de Atenção Básica. Entre os objetivos que caracterizam o Programa Saúde na Escola encontra-se a de promover a saúde de crianças e adolescentes a partir da conscientização de aspectos e fatores que influenciam na qualidade de vida dos indivíduos incentivando a prática de hábitos saudáveis e o conhecimento de possíveis comportamentos de risco à saúde.

Com o objetivo de ampliar a compreensão do processo de implementação do PSE o artigo apresenta as mudanças nas ações de saúde voltadas às crianças e adolescentes de um modelo focado na cura para uma abordagem centrada na promoção de saúde e em como essa mudança de paradigma influenciou a forma de implementação das políticas públicas de promoção da saúde às crianças e adolescentes.

O artigo está organizado com esta introdução e mais 02 partes. Na próxima seção será destinada a compreensão da trajetória das políticas públicas de saúde, com o foco nas abordagens nas quais as ações em saúde se basearam. O objetivo é de analisar como os preceitos de promoção da saúde modificaram as ações de implementação das políticas públicas de saúde.

Na segunda seção será analisado o Programa Saúde na Escola na perspectiva de um programa intersetorial de promoção da saúde no ambiente escolar. Será visto também como as ações de saúde escolar têm a capacidade de mobilizar atores e instituições governamentais e não governamentais em torno desse tema.

\section{PROMOÇÃO DA SAÚDE: RUPTURA E INTERSETORIALIDADE}

O conceito promoção da saúde se baseia num processo de quebra de valores e práticas na área da saúde que tiveram longo histórico nos modelos de políticas públicas de saúde no Brasil.

A trajetória das políticas públicas de saúde no Brasil durante o século XX demonstra que as ações e práticas de saúde estiveram fundamentadas em perspectiva curativa, individual, baseada na especialização médica e centradas em hospitais como lócus privilegiados dos serviços de saúde.

No período de 1880 a 1930, refere-se ao período na qual o Brasil está na fase de organização da estrutura dos serviços de saúde no âmbito do Estado. Com isso os serviços foram estabelecidos em 
uma relação vertical, com imposição de normas e segregação social, desconsiderando o conhecimento popular, principalmente dos pobres e dos negros, e privilegiando o saber científico na qual os técnicos de saúde tinham domínio. A partir de 1964 o Brasil adota o modelo de saúde baseado em grandes hospitais, que coloca em segundo plano a rede de atenção básica e, com isso, houve grandes mudanças na área da saúde no País. O governo coloca em primeiro plano o investimento nos procedimentos de alta complexidade e, portanto, de alto custo. Nesse contexto, o governo favoreceu o modelo de saúde curativa e especializada. A ação estava voltada para os hospitais e ambulatórios. (SARRETA, 2009)

No âmbito das ações de saúde nas escolas o modelo desenvolvido durante o século XX se concretizou em práticas higienistas que tinham como objetivo central a melhoria das condições sanitárias da sociedade. A escola passou a ser considerado um espaço privilegiado para o desenvolvimento de práticas direcionadas à melhoria das condições sanitárias de uma parcela significativa da população, as crianças, com ênfase na higiene, tanto física como dos modos de ser. (BIZZO; MONTEIRO, 2015)

Nesse sentido, embora fosse uma prática de saúde em um contexto escolar, ainda não verifica uma prática intersetorial entre saúde e educação e sim uma prática setorial da área da saúde que utiliza o espaço escolar como uma expansão local das práticas que também ocorriam nos hospitais e demais instituições de saúde.

As práticas de saúde escolar mais freqüentes se fundamentam no paradigma tradicional de saúde escolar, que consiste em intervenções do setor saúde no âmbito escolar. Por exemplo, vacinação, desparasitação, controles médicos, ou palestras de profissionais da saúde nas escolas. Os professores também realizam múltiplas ações nesta linha, como controle do cartão de vacinas, participação em campanhas de prevenção, ações de detecção e encaminhamento de problemas de saúde. Dentro deste enfoque, predomina a lógica biomédica, centrada na prevenção da doença. A escola envolvida geralmente tem uma posição passiva nestas atividades, pois se concebe como um cenário para as ações de saúde.

Ainda de acordo com os pesquisadores Paulo Henrique Nico Monteiro e Nelio Bizzo (2014) o conhecimento da área da saúde serviu para regulamentar, estruturar e direcionar as práticas educacionais.

Baseada em preceitos originários da área da saúde e a partir de sua racionalidade científica, fundamentada na fisiologia, a "saúde escolar" começa a fazer parte do ambiente da escola e passa a definir ações e intervenções durante todo o século XX. Aspectos relacionados à arquitetura escolar, à infraestrutura, à disposição e às especificações do mobiliário, à regulamentação e à orientação nutricional da merenda escolar, aos exames antropométricos e à detecção de possíveis agravos ou deficiências na população escolar faziam parte desse tipo de ação. (BIZZO; MONTEIRO, 2015).

Verifica, nessa trajetória das políticas públicas de saúde, que os preceitos do individualismo, da especialização e do curativismo compuseram a estrutura ideológica dominante na área da saúde e, por consequência, as práticas de saúde em ambiente escolar em saúde foram fortemente influenciadas por esses preceitos.

O período de redemocratização do país e a promulgação da Constituição de 1988 foi o início de ruptura desses paradigmas com a adoção dos princípios da promoção da saúde. Conforme nos aponta Lucíola Santos Rabello a promoção da saúde constitui-se: 
um novo paradigma em saúde, da incorporação dos conteúdos sociais nesta abordagem, como uma tentativa efetiva dos atores sociais de ampliarem a apropriação da interdisciplinaridade em seu processo de trabalho cotidiano, possibilita a compreensão de que o tema da saúde é social e não somente uma questão técnica e está diretamente relacionado com a forma de sociedade e de Estado. (RABELLO, 2010).

O conceito de promoção de saúde não só amplia o escopo de ação da área da saúde, mas estimula a inclusão de novos atores, instituições e a interação entre políticas públicas de outras áreas que, a partir de então, se apropriam dos temas da área da saúde.

Com isso houve um movimento internacional que fomentou encontros em forma de congressos, assembléias que culminaram na construção da compreensão do conceito de promoção da saúde e à percepção ampliada de saúde como um fator também social. Um dos documentos produzidos nesses encontros foi a Carta de Ottawa onde afirma-se: "A política de promoção da saúde requer a identificação e a remoção de obstáculos para a adoção de políticas públicas saudáveis nos setores que não estão diretamente ligados à saúde". (OTTAWA, 1986)

Essas mobilizações e os documentos produzidos em torno dos princípios de promoção da saúde possibilitaram a perspectiva de estender as práticas de saúde para além dos locais habituais, tais como clínicas e hospitais, somando os conhecimentos consolidados de sua área com as práticas sociais e o diálogo entre diversos atores da sociedade organizada e instituições governamentais com os profissionais da saúde. Nessa perspectiva, foi possível organizar práticas intersetoriais das políticas de saúde, promovendo a integração de ações como é o caso das políticas de educação e saúde.

As Escolas Promotoras de Saúde que surgiram no final da década de 80 do século XX tiveram um papel central nas primeiras práticas de promoção da saúde em contexto escolar. Nesse modelo, os integrantes da instituição educativa, do setor saúde e da comunidade demandaram ações protagonistas na identificação das necessidades e problemas de saúde e na definição de estratégias e linhas de ação pertinentes para abordá-los e enfrentá-los. (SHEPHERD, 2003).

Portanto, pode-se reconhecer que houve nas Escolas Promotoras de Saúde uma primeira prática genuinamente intersetorial, possibilitada pela adoção da promoção da saúde.

Com isso, a área da educação envolve-se nessa concepção de promoção da saúde onde a escola constitui-se num local privilegiado. Segundo as pesquisadoras Camília Susana Faler e Maria Isabel Barros Bellini:

Reitera-se que a escola é um importante espaço para o cuidado, a proteção, a promoção da saúde, o exercício da cidadania e a inclusão social, lócus que possibilita a viabilização de informação, reflexão e promoção das políticas de educação e saúde. Portanto, espaço fundamental para implementação de ações que contribuam para o exercício de cidadania dos adolescentes, para a diminuição dos custos do sistema de saúde e para consecução de uma educação integral. (BELLINI; FALER, 2014).

Ao conceber as práticas de ação de saúde no contexto escolar, na ótica da promoção da saúde, constrói-se uma narrativa na atuação da área da saúde e da educação que amplia a finalidade de ambas as áreas para além da saúde exclusivamente "curativa" e uma educação exclusivamente "conteudista", compreendendo a educação para a saúde com enfoque integral onde

Incorpora a Educação para Saúde em nível curricular e como parte do projeto institucional. Utiliza todas as oportunidades educativas em nível formal e informal para promover a saúde. Promove a reflexão e a análise crítica da informação; facilita a 
conscientização e o "empoderamento" dos estudantes e da comunidade educativa como um todo. Busca desenvolver novos conhecimentos e habilidades que contribuam para a adoção e manutenção de estilos de vida saudáveis, por meio de técnicas participativas e atividades significativas que possam transcender o âmbito escolar. (SHEPHERD, 2003).

Na perspectiva da área da saúde "promover saúde em um espaço diferenciado dos espaços tradicionais em que essa política é debatida, o processo de pensar saúde no espaço da escola propicia ampliar os olhares e rompe com uma perspectiva histórica centrada na doença, na medicalização, no modelo hospitalocêntrico". (BELLINI, FALER, 2014)

As abordagens de promoção da saúde possibilitaram uma mudança no entendimento do que deve ser tratado pelas políticas de saúde voltadas às crianças e adolescentes quando se passou de um entendimento focado na "cura" para uma abordagem centrada na promoção da saúde e ainda sugerem que a adoção do conceito de promoção da saúde com foco na criança e adolescentes favorece ações intersetoriais entre a saúde e a educação. Essa abordagem aparece em políticas públicas intersetoriais de saúde e educação, tal como o Programa Saúde na Escola.

\section{PROGRAMA SAÚDE NA ESCOLA}

Neste trabalho foi realizada uma pesquisa-ação com alunos da $4^{a}$ etapa da Educação de Jovens e 0 Programa Saúde na Escola (PSE), instituído pelo decreto presidencial n⿳0 6.286, de 05 de dezembro de 2007, integra ações entre o Ministério da Saúde e o Ministério da Educação e compõe o Plano Nacional de Atenção Básica, plano este pertencente às ações do Ministério da Saúde, acompanhado e coordenado pelo Departamento de Atenção Básica.

Entre os objetivos que caracterizam o Programa Saúde na Escola encontra-se a de promover a saúde de crianças e adolescentes, a partir da conscientização de aspectos e fatores que influenciam a qualidade de vida dos indivíduos, incentivando a prática de hábitos saudáveis e o conhecimento de possíveis comportamentos de risco à saúde.

O PSE pode ser considerado uma política social que está vocacionalmente ligado ao Programa Estratégia Saúde na Família. [...] a concepção inerente à ESF traz uma visão ampliada de saúde que a relaciona a diversos fatores que determinam as condições de saúde e de qualidade de vida dos sujeitos nos seus diferentes contextos, tais como: sociais, econômicos, ambientais, psicológicos e políticos, entre outros, representando, assim, uma prática inovadora de atenção à saúde (VIEIRA, 2013, p. 56).

As ações promovidas pelo PSE vinculam-se às mudanças de paradigmas na área da saúde enquanto ampliação do "espaço" aonde as ações de saúde devem ocorrer e consequentemente à participação de novos atores na produção de políticas públicas de saúde, especialmente na sua implementação.

O PSE parte do princípio de que as questões relativas à promoção da saúde devem ser desenvolvidas para além dos serviços, uma vez que as práticas de promoção da saúde inserem-se em diferentes espaços sociais, a exemplo das escolas (MS, MEC, 2011). Nesse contexto aponta Vieira (2013): Identifica-se, a partir dele, uma interseção que pode ser estratégica para a gestão das ações da saúde e de revisão da prática assistencial, no que se refere à necessidade de alianças e parcerias com outros setores, incluindo o da educação. 
Para avançar nessa direção, a Política de Atenção Básica em Saúde e a Política de Educação Básica investem na universalização do Programa Saúde na Escola - PSE na perspectiva de uma conexão entre esses setores para ampliação das ações intersetoriais de promoção à saúde, prevenção de agravos e doenças e de cuidado à saúde das crianças, adolescentes e jovens, longitudinalmente, num processo compartilhado de gestão (VIEIRA, 2013, p. 42).

O PSE como uma política pública intersetorial associada às políticas de atenção básica à saúde, cumpre a função social de promover a ações de saúde em ambiente escolares para crianças e adolescentes baseando-se no conceito de promoção da saúde.

Por esse ângulo as ações promovidas por este programa vinculam-se às mudanças de paradigmas na área da saúde quanto à ampliação do espaço onde as ações de saúde devem ocorrer; a compreensão da atenção básica à saúde vinculada à promoção da saúde; a valorização dos conhecimentos e práticas sociais em saúde e não somente o conhecimento técnico da área e; a participação de novos atores na produção de políticas públicas de saúde, especialmente a sua implementação.

Para analisarmos tanto as questões dos princípios da promoção da saúde quanto da intersetorialidade no PSE será examinado partes do documento: "Passo a Passo PSE Programa Saúde na Escola Tecendo caminhos da intersetorialidade"; produzido de forma conjunta pelos Ministérios da Saúde e da Educação em 2011.

Já em sua introdução o documento faz importante ressalva que serve para observarmos a diferença que há entre um modelo tradicional de ação de saúde nas escolas de um modelo que tem a promoção da saúde como base: "É preciso compreender que o espaço escolar não deve ser utilizado para consultas médicas com o objetivo da medicalização ou de diagnóstico clínico-psíquico dos fracassos do processo ensino-aprendizagem, mas apenas para triagens, por sua objetividade e ganho de escala em ambiente coletivo" (MS, MEC, 2011)

Portanto, as atividades de saúde em ambiente escolar a partir desse Programa visam que crianças e adolescentes tenham uma primeira via de acesso a serviços de saúde, que não compreende os serviços de medicalização e de diagnóstico, mas, antes, de orientação e encaminhamento.

Outro destaque é a integração do conhecimento local, o conhecimento muitas vezes construído na comunidade na qual tanto a escola e a instituição de saúde se encontram. Esse reconhecimento é um dos princípios da promoção da saúde na qual incentiva

Nas escolas, o trabalho de promoção da saúde com os educandos, e também com professores e funcionários, precisa ter como ponto de partida "o que eles sabem" e "o que eles podem fazer". É preciso desenvolver em cada um a capacidade de interpretar o cotidiano e atuar de modo a incorporar atitudes e/ou comportamentos adequados para a melhoria da qualidade de vida. Desse modo, profissionais de saúde e de educação devem assumir uma atitude permanente de emponderamento dos princípios básicos de promoção da saúde por parte dos educandos, professores e funcionários das escolas. (MS, MEC, 2011)

O documento ainda elenca as diretrizes do PSE onde pode-se perceber os preceitos da promoção da saúde. Portanto, são destacados

Promover a articulação de saberes, a participação dos educandos, pais, comunidade escolar e sociedade em geral na construção e controle social das políticas públicas da saúde e educação; fortalecer o enfrentamento das vulnerabilidades, no campo da saúde, que possam comprometer o pleno desenvolvimento escolar; promover a 
comunicação, encaminhamento e resolutividade entre escolas e unidades de saúde, assegurando as ações de atenção e cuidado sobre as condições de saúde dos estudantes; atuar, efetivamente, na reorientação dos serviços de saúde para além de suas responsabilidades técnicas no atendimento clínico, para oferecer uma atenção básica e integral aos educandos e à comunidade. (MS; MEC, 2011).

De fato, as ações que o PSE implementam tem como base a promoção da saúde e, dessa forma, fomenta a coordenação intersetorial entre saúde e educação, além de incentivar a participação ativa da comunidade na resolução dos problema de saúde local. Nesse contexto aponta Vieira (2013)

\begin{abstract}
"identifica-se, a partir dele, uma interseção que pode ser estratégica para a gestão das ações da saúde e de revisão da prática assistencial, no que se refere à necessidade de alianças e parcerias com outros setores, incluindo o da educação (...) na perspectiva de uma conexão entre esses setores para ampliação das ações intersetoriais de promoção à saúde, prevenção de agravos e doenças e de cuidado à saúde das crianças, adolescentes e jovens, longitudinalmente, num processo compartilhado de gestão (VIEIRA, 2013).
\end{abstract}

O documento elenca duas diretrizes para as ações de coordenação intersetorial do PSE, a saber: articular as ações do Sistema Único de Saúde (SUS) às ações das redes de educação pública de forma a ampliar o alcance e o impacto de suas ações relativas aos educandos e suas famílias, otimizando a utilização dos espaços, equipamentos e recursos disponíveis e; promover a comunicação, encaminhamento e resolutividade entre escolas e unidades de saúde, assegurando as ações de atenção e cuidado sobre as condições de saúde dos estudantes. (MS; MEC, 2011).

Essas diretrizes apontam um modelo de gestão que promove as ações de coordenação no processo de implementação de uma política pública intersetorial. Visando a organização das ações intersetoriais do programa foi constituído o Grupo de Trabalho Intersetorial, que é organizado obedecendo ao sistema federativo na qual o Estado está alicerçado.

Para compreendermos as ações de coordenação intersetorial do PSE há nesse documento a apresentação dos Grupos de Trabalho Intersetorial (GTI) nas três esferas federativas: federal, estadual e municipal que são a base da implementação intersetorial do PSE.

Dessa forma, o grupo de cada esfera federativa tem atribuições diferenciadas.O grupo de trabalho intersetorial federal (GTI-F) tem a prerrogativa de coordenar, promover e subsidiar as ações nos estados e municípios provendo materiais de apoio à implementação e avaliação do programa, calendário de ações nacionais de saúde, interlocução entre entidades nacionais da área da Saúde e Educação como a Conselho Nacional de Secretários de Saúde (Conass), Conselho Nacional de Secretaria Municipais de Saúde (Conasems), Conselho Nacional de Secretários de Educação (Consed), União dos Dirigentes Municipais de Educação (Undime).

No âmbito dos estados há o grupo de trabalho intersetorial estadual (GTI-E), que tem por finalidade apoiar institucionalmente os municípios e mobilizá-los para adesão ao programa definindo estratégia de ação em cada município e apoiando o gestor municipal na articulação, planejamento e implementação das ações do PSE; outra atribuição do GTI-E é articular as redes de saúde para as ações de PSE e Pactuar, nas Comissões Intergestores Bipartites (CIB) do Sistema Único de Saúde, encaminhamentos e deliberações no âmbito do PSE, conforme fluxo de adesão.

O grupo de trabalho intersetorial municipal (GTI-M) busca uma maior capilariedade e articulação com o território, oferecendo apoio às unidades de saúde e unidades educacionais na associação de ações e definindo estratégias às ações. 
De forma geral, o documento indica que o trabalho no GTI pressupõe, dessa forma, interação com troca de saberes, poderes e afetos entre profissionais da saúde e da educação, educandos, comunidade e demais redes sociais (MS, MEC, 2011).

Com essa proposta os participantes de cada GTI variam conforme o grupo que participa, possibilitando a atuação de diversos atores interessados no tema de saúde escolar.

[...] os GTIs devem ser compostos, obrigatoriamente, por representantes das Secretarias de Saúde e de Educação e, facultativamente, por outros parceiros locais representantes de políticas e movimentos sociais (cultura, lazer, esporte, transporte, planejamento urbano, sociedade civil, setor não governamental e setor privado, entre outros) (MS, MEC, 2011, p. 12).

A partir dessa lógica de ação no território, onde há ações capilarizadas e articuladas na comunidade em que o PSE está atuando, o GTI-M funciona como um aglutinador desses atores e instituições em torno da temática de saúde escolar.

As dissertações e teses elaboradas nas universidades brasileiras que tiveram como tema de estudo o PSE e a sua intersetorialidade ajudam a compreender o Programa as particularidades da implementação dessa política pública.

A pesquisa realizada em 2014 por Isabelle Carolline Verossimo Farias no município de Olinda-PE buscou observar os atores que se articulavam para a implementação do PSE no município. Segundo as informações do estudo "em 2013, Olinda aderiu ao PSE com um total de 43 escolas (41 escolas e 02 creches) e vinculou 35 Estratégias de Saúde da Família, totalizando uma cobertura de 13.562 alunos da rede pública de ensino" (FARIAS, 2014, p. 44).

A pesquisa analisou as vinte primeiras escolas que aderiram ao Programa. No município de Olinda a pesquisadora aponta que houve mobilização das Secretarias Municipal de Educação, Saúde, Cultura e Ação Social, além de parcerias com entidades não governamentais. De acordo com a autora: Em relação aos outros setores governamentais que se envolvem intersetorialmente nas ações que o PSE desenvolvidas no município de Olinda além da Saúde e Educação, foram citados a Secretaria do Esporte e da Cultura e a Secretaria de Ação Social e setores não-governamentais como Premetropole, Banco Santander e igreja local (FARIAS, 2014, p .45).

De modo semelhante, a participação de empresas é relatada na pesquisa de Fernanda Bressan no estudo de caso do município de Blumenau. Nesse caso é interessante notar que a parceria com empresas inclui o fornecimento de artigos da área da saúde para as crianças atendidas pelo PSE, como no caso narrado pelo estudo: "na Secretaria Municipal de Educação, havia uma parceria com o Lions e por intermédio deles conseguíamos os óculos para as crianças" (BRESSAN, 2014, p. 42). Consequentemente havia a participação de inúmeros agentes na efetivação das ações do PSE em Blumenau, que conforme o relato da pesquisa foi incentivado pela própria instrução do programa, ou seja, as orientações que vinham do Ministério da Saúde e Educação.

Destacam-se que, segundo descrevem as instruções do Programa, têm participação prevista no desenvolvimento do PSE a sociedade civil, empresas privadas, movimentos sociais e outros segmentos governamentais, como a Secretaria de Cultura, Esporte e Lazer e Transporte, devendo, inclusive, essas parcerias serem encorajadas (BRESSAN, 2014, p. 55). 
Outra estratégia de estabelecer parcerias é o caso adotado pela Prefeitura do Rio de Janeiro para transferir à iniciativa privada a implementação do PSE, segundo a pesquisa realizada na região de Manguinhos. Estratégias acionadas para se implementar o PSE em Manguinhos: a gestão privada do Estado.

Dentro desse contexto, se efetivou essa parceria entre o setor público e a iniciativa privada dentro das ações de políticas públicas do município. No caso do Rio de Janeiro, para realizar ações de saúde na escola, a viabilização do programa somente se tornou possível mediante a ação da iniciativa privada, ou seja, a Prefeitura da Cidade do Rio de Janeiro modifica sua ação estatal na medida em que contrata organizações sociais para implantar políticas públicas que são de sua responsabilidade (BRANDÃO, 2014, p. 65).

Contudo, em alguns municípios demonstra-se que havia atores que implementavam ações de saúde escolar antes da instituição do PSE, ou seja, há outras políticas públicas de saúde escolar que também mobilizaram atores e instituições e que o PSE ocasionalmente se beneficiou. Essa questão fica claro quando analisamos o município de Goiânia.

\begin{abstract}
"Antes de 2008, a gente já tinha um trabalho de articulação entre saúde e educação, com outros nomes: "Educação Promotora de Saúde; e de outros nomes" e que quando chegou o PSE, não foi difícil vislumbrar o que era todo um desejo que tanto a saúde como a educação tinha de institucionalizar, para que a gente pudesse ter governabilidade entre os dois setores e principalmente recursos. Então, logo que foi instituída a portaria, em 2009, nós começamos a articulação e conseguimos efetivar esse grupo gestor através desse decreto interministerial (GTI municipal)" (MOURA, 2014).
\end{abstract}

Podemos compreender que o objetivo do Governo Federal em induzir políticas de saúde escolar nos municípios a partir de um programa estruturado foi possível a partir da mobilização de rede de atores e instituições preexistente ao PSE que se interessavam pelo tema de saúde escolar. Em alguns municípios, como Goiânia, houve uma adequação desta rede para atender as especificidades do PSE.

Bom, com a criação do PSE, que foi via decreto presidencial, no final de 2008, chamouse todas as pessoas que tinham militância nessa área para se pensar uma forma de trabalhar o PSE, já que ele surgiu como uma política de articulação intersetorial. Para viabilizar essa política, teria que criar um grupo gestor porque o PSE cobrava isso em seu decreto presidencial, cobrava uma equipe gestora contemplando principalmente saúde e educação (teve outros setores que foram envolvidos) pra fazer a primeira gestão do PSE". (MOURA, 2014).

Portanto, a variedade de estratégias encontradas por cada município para implementação do PSE está na forma em como as parcerias e as redes se dão e se formam e quais são os atores e instituições que participam de sua implementação a partir da estruturação dos Grupos de Trabalho Intersetorial.

De qualquer forma há o predomínio da participação das Secretarias Municipais de Saúde e Educação que funcionam como estrutura básica para os demais participantes poderem realizar as ações do PSE em conjunto com estas secretarias, de forma que a participação de outros atores e instituições é incentivada pelo Ministério da Saúde e Educação para se ter uma implementação de ações de promoção da saúde mais efetiva nas escolas.

Evidencia-se, a partir da leitura desses estudos e dos documentos institucionais dos Ministérios da Saúde e Educação, que a intersetorialidade e os princípios de promoção da saúde estão efetivamente 
presentes na implementação do PSE. Os questionamentos da participação de novos atores e novas instituições permitem que possamos aprofundar a análise da implementação de políticas públicas de saúde escolar, considerando as mudanças ocorridas a partir da adoção da intersetorialidade por estas políticas públicas.

\section{CONCLUSÃO}

As políticas públicas intersetoriais para a promoção da saúde da criança e do adolescente envolvem a necessidade de coordenar e articular atores diversos e distintas estruturas burocráticas e instituições. Nessa implementação intersetorial há a quebra de paradigma da organização setorial das Secretarias de Educação e Saúde promovendo a participação de diversos atores, o que requer uma estrutura que garanta a articulação e também o compartilhamento de ações, de funções, de recursos. Esse processo possibilita a ampliação na interlocução e interação entre atores e instituições.

As atividades intersetoriais de saúde e educação no âmbito da promoção da saúde permitem que, de fato, não somente a perspectiva medicamentosa ou curativista conduzem as ações de saúde em contexto escolar, mas os aspectos educacionais entrelaçados ao contexto social, cultural e educacional encontram também espaço de ações de saúde nas escolas.

Dentre às políticas públicas que adotam a perspectiva intersetorial de promoção da saúde no ambiente escolar há o Programa de Saúde na Escola. A implementação deste programa estruturou os Grupos de Trabalho Intersetorial em âmbito federal, estadual e municipal, como uma prática que deve contribuir para romper a estrutura burocrática setorial e estabelecer novas formas de implementação intersetorial a partir, principalmente, da articulação entre as estruturas administrativas e os atores.

Podemos observar que, ao adotar os princípios da promoção da saúde e a lógica da intersetoridade no processo de implementação, o Programa Saúde na Escola traz desafios para sua implementação eficaz, entre eles a complexidade existente nas estruturas burocráticas que, em si, não foram planejadas para que haja ações intersetoriais e que facilitem a comunicação entre os atores.

Este artigo ao reunir evidências que demonstram que houve mudanças significativas na abordagem nas ações de saúde busca contribuir para o debate sobre o desenvolvimento das políticas públicas de promoção à saúde de crianças e adolescentes e nos desafios na implementação de políticas intersetoriais de saúde e educação.

\section{REFERÊNCIAS}

ANSELL, Chris; GASH, Allison. Collaborative Governance in Theory and Practice. Journal of Public Administration Research and Theory. Oxford University Press. Oxford, 2007.p. 543-571.

BELLINI, Maria Isabel Barros; REHERMANN, Vanessa Soares; MORÉS, Fernanda Brenner. Aprender saúde na escola: uma experiência de intersetorialidade entre as políticas de saúde e educação. In: BELLINI, Maria Isabel Barros; FALLER, Camília Susana. Intersetorialidade e políticas sociais. Editora PUC-RS. Porto Alegre, 2010. 228p.

MONTEIRO, Paulo Henrique Nico; BIZZO, Nelio. A saúde na escola: análise dos documentos de referência nos quarenta anos de obrigatoriedade dos programas de saúde, 19712011. Hist. cienc. saude-Manguinhos, Rio de Janeiro, v. 22, n. 2, p. 411- 
428, jun. 2015. Disponível em <http://www.scielo.br/scielo.php?script=sci_arttext\&pid=S010459702015000200007\&lng=pt\&nrm=iso >. acessos em 22 nov. 2019. Epub 19-Dez2014. http://dx.doi.org/10.1590/S0104-59702014005000028.

BRASIL. Passo a Passo PSE: Construindo os caminhos da intersetorialidade. Ministério da Saúde, Ministério da Educação. Brasília. 2011. 48p.

BRANDÃO, Roberto Duarte Albino. A implementação do Programa Saúde na Escola no Território de Manguinhos - RJ: estratégias de privatização na saúde pública. 2014. 144p. Dissertação. Mestrado Profissional em Educação Profissional em Saúde. Fundação Oswaldo Cruz.

BRESSAN, Fernanda. O Programa Saúde na Escola: interfaces entre saúde e educação no município de Blumenau-SC. 2014. 78p. Dissertação. Mestrado em Educação. Universidade Regional de Blumenau, Faculdade de Educação.

FARIAS, Isabelle Carolline Verossimo de. Análise da intersetorialidade no Programa Saúde na Escola no município de Olinda-PE. 2014. 99p. Dissertação. Mestrado em Saúde Coletiva. Universidade Federal de Pernambuco.

INSTITUTO BRASILEIRO DE GEOGRAFIA E ESTATÍSITCA. Pesquisa Brasileira de Saúde Escolar - 2015. Rio de Janeiro: IBGE, 2016. 132 p.

MOURA, Samara Marques de. Programa Saúde na Escola: um instrumento da intersetorialidade na promoção da saúde. 2014. 108p. Dissertação. Mestrado em Odontologia. Universidade Federal de Goiás.

OTTAWA. Charter for Health Promotion. World Health Organization. Ottawa, 1986.

RABELLO, Lucíola Santos. Promoção da saúde: a construção social de um conceito em perspectiva comparada do SUS. Rio de Janeiro: Editora FIOCRUZ, 2010. 228 p.

SARRETA, FO. Educação permanente em saúde para os trabalhadores do SUS [online]. São Paulo: Editora UNESP; São Paulo: Cultura Acadêmica, 2009. 248 p. ISBN 978-85-7983-009-9. Available from SciELO

SCHERER, M. D. A. et al. Ruptures and resolutions in the health care model: reflections on the Family Health Strategy based on Kuhn's categories, Interface - Comunic., Saúde, Educ., v.9, n.16, p.53-66, set.2004/fev.2005.

SILVA, Carlos dos Santos. Escola Promotora de Saúde: uma Visão Crítica da Saúde Escolar. In: Sociedade Brasileira de Pediatria. Escola promotora de saúde. Brasília: Sociedade Brasileira de Pediatria; 2003

VIEIRA, Maria Edna Moura. Programa Saúde na Escola: a intersetorialidade em movimento. 2013. 94f. Dissertação. Mestrado em Psicologia. Universidade de Brasília, Instituto de Psicologia.

WORLD HEALTH ORGANIZATION. Growing up unequal: gender and socioeconomic differences in young people's health and well-being: health behaviour in school-aged children (HSBC) study - international report from the 2013/2014 survey. Dinamarca. 2016. 275p.

Policies: Structures, actions and experiences. WHO Regional Office for Europe. Copenhagen, 2012. 228p.

Submissão: 08/08/2021 